\title{
Validação do Modelo KiBaM para Predição do Tempo de Vida de Baterias Utilizadas em Dispositivos Móveis
}

\author{
Douglas Joziel Bitencourt Freitas ${ }^{1}$ \\ Paulo Sérgio Sausen ${ }^{2}$ \\ Airam Tereza Zago Romcy Sausen ${ }^{3}$ \\ Manuel Martin Pérez Reimbold ${ }^{4}$ \\ Programa de Pós-Graduação Stricto Sensu em Modelagem Matemática, UNIJUÍ, Ijuí, RS
}

Resumo. Neste artigo é apresentada a validação do Modelo KiBaM para predição do tempo de vida de baterias utilizadas em dispositivos móveis. O modelo foi validado através da comparação entre os resultados das simulações computacionais e dados experimentais de baterias de Li-Po, sendo que para descargas variáveis, o erro médio foi de 1,84\%.

Palavras-chave. Modelagem Matemática, Modelo KiBaM, Simulação Computacional

\section{Introdução}

As baterias eletroquímicas consagraram-se como a tecnologia de armazenamento portátil de energia mais antiga e ainda são largamente utilizadas em diversas aplicações. O desenvolvimento de novos tipos de baterias e, principalmente, a miniaturização para atender demandas específicas de energia, fomentou o avanço da indústria para produção em larga escala de dispositivos móveis - smartphones, celulares, tablets, etc. - cada vez mais compactos. Os dispositivos móveis agregam mobilidade, comodidade e facilidade de acesso às redes de voz e dados, tornando-se acessório popular e indispensável para comunicação, trabalho, estudo e, até mesmo, lazer.

As baterias recarregáveis, utilizadas em dispositivos móveis, têm capacidade finita para armazenamento de energia, necessitando de uma recarga a cada período de uso. Pode-se concluir, então, que o tempo operacional de um determinado dispositivo, está limitado à duração da fonte de energia (i.e., tempo de vida da bateria). Diante disto, julga-se importante dispor de métodos para predizer o tempo de vida de baterias e, consequentemente, o tempo de funcionamento dos dispositivos. Na literatura técnica, um dos métodos empregados é a modelagem matemática, através de modelos que descrevem a dinâmica da descarga de baterias, a partir das características físicas ou de um conjunto de dados experimentais.

\footnotetext{
${ }^{1}$ douglasjoziel@outlook.com

${ }^{2}$ sausen@unijui.edu.br

3 airam@unijui.edu.br

${ }^{4}$ manolo@unijui.edu.br
} 
Vários modelos matemáticos que descrevem a descarga de baterias vêm sendo desenvolvidos, dos quais pode-se destacar os modelos eletroquímicos [1], elétricos [2], estocásticos [3], analíticos [4], via Identificação de Sistemas [5] e, mais recentemente, híbridos [6]. Da classe dos modelos analíticos, o Modelo Cinético de Bateria (KiBaM) utiliza como base de seu fundamento matemático a cinética, modelando a velocidade das reações químicas do processo e a influência de fatores acessórios [7]. Um estudo comparativo entre as simulações do Modelo KiBaM e os resultados do programa DUALFOIL ${ }^{5}$ [8], aponta erro inferior a $5 \%$ para correntes contínuas e variáveis [9]. Outro estudo, comparando as simulações do Modelo KiBaM com dados experimentais de baterias de Lítio-Íon Polímero (Li-Po), indica um erro médio de $1,07 \%$ para correntes contínuas [10].

Neste contexto, o Grupo de Automação Industrial e Controle (GAIC), da Universidade Regional do Noroeste do Estado do Rio Grande do Sul (UNIJUÍ), tem realizado diversas pesquisas, no intuito de verificar qual o modelo matemático mais adequado para predizer o tempo de vida de baterias utilizadas em dispositivos móveis, sob diferentes especificações [11]. Sendo assim, o principal objetivo deste trabalho é apresentar os resultados de validação do Modelo KiBaM, utilizado para modelar a descarga de baterias de Li-Po, sob perfis de corretes variáveis, visando consolidá-lo como substituto simples de fácil usabilidade e implementação, de boa acurácia e à altura de modelos mais complexos (e.g. eletroquímicos e estocásticos).

O artigo está organizado conforme segue. Na Seção 2 são apresentadas generalidades sobre o Modelo KiBaM, bem como, a resolução das equações. Na Seção 3 é apresentada de modo breve a metodologia empregada para coleta de dados e estimação dos parâmetros do modelo - neste caso, utilizando o método dos Mínimos Quadrados (MQ). Na Seção 4 são discutidos os resultados das simulações, através de análises comparativas. E, por fim, na Seção 5, considerações finais e perspectivas para os trabalhos futuros.

\section{Modelagem Matemática}

Nesta seção é apresentado os aspectos gerais do Modelo KiBaM e a resolução de suas equações. Na prática, o processo de descarga de uma bateria tem características e efeitos não lineares - nível de cutoff, efeito de recuperação, taxa de capacidade, etc. - que interferem diretamente no tempo de vida [9]. O Modelo KiBaM captura dois desses principais efeitos: taxa de capacidade e recuperação.

O Modelo KiBaM considera a carga da bateria distribuída em duas fontes: a fonte de carga disponível e a fonte de carga limitada (Figura 1). Observa-se que uma fração $c$ da capacidade total é distribuída na fonte de carga disponível, e uma fração $1-c$ na fonte de carga limitada. A fonte de carga disponível fornece elétrons diretamente a corrente $i(t)$, enquanto a fonte de carga limitada fornece elétrons à de carga disponível - o parâmetro $k$ é a razão de fluxo entre as fontes [7].

Considerando $i(t)=I$ uma corrente constante, as equações que descrevem o modelo

\footnotetext{
${ }^{5}$ Programa que simula descarga de baterias, baseado em um modelo eletroquímico.
} 


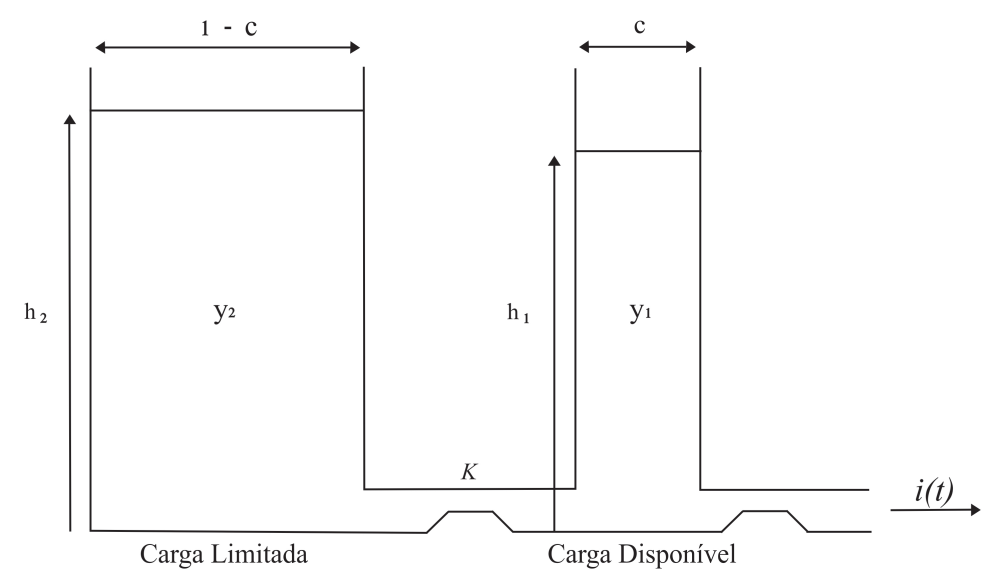

Figura 1: Esquema de representação do Modelo KiBaM [7].

são as seguintes:

$$
\begin{aligned}
& \frac{d y_{1}(t)}{d t}=-I-k\left(h_{1}(t)-h_{2}(t)\right) \\
& \frac{d y_{2}(t)}{d t}=k\left(h_{1}(t)-h_{2}(t)\right),
\end{aligned}
$$

onde: $y_{1}(t)$ é a carga disponível e $y_{2}(t)$ a carga limitada. A altura em cada um dos tanques é dada por

$$
\begin{aligned}
h_{1}(t) & =\frac{y_{1}(t)}{c} \\
h_{2}(t) & =\frac{y_{2}(t)}{1-c} .
\end{aligned}
$$

Por simplificação matemática, uma nova razão constante $k^{\prime}$ é definida como

$$
k^{\prime}=\frac{k}{c(1-c)} .
$$

Substituindo as equações (3), (4) e (5) nas equações (1) e (2) tem-se

$$
\begin{aligned}
& \frac{d y_{1}(t)}{d t}=-I-k^{\prime}(1-c) y_{1}(t)+k^{\prime} c y_{2}(t) \\
& \frac{d y_{2}(t)}{d t}=k^{\prime}(1-c) y_{1}(t)-k^{\prime} c-y_{2}(t) .
\end{aligned}
$$

As equações (6) e (7) podem ser resolvidas aplicando-se as definições da Transformada de 
Laplace, obtendo-se como resultado final

$$
\begin{aligned}
& y_{1}(t)=y_{1}(0) e^{-k^{\prime} t}+\frac{\left(y_{0} k^{\prime} c-I\right)\left(1-e^{-k^{\prime} t}\right)}{k^{\prime}}-\frac{I c\left(k^{\prime} t-1+e^{-k^{\prime} t}\right)}{k^{\prime}} \\
& y_{2}(t)=y_{2}(0) e^{-k^{\prime} t}+y_{0}(1-c)\left(1-e^{-k^{\prime} t}\right)-\frac{I(1-c)\left(k^{\prime} t-1+e^{-k^{\prime} t}\right)}{k^{\prime}},
\end{aligned}
$$

onde: $y_{1}(0)$ e $y_{2}(0)$ são a quantidade de carga disponível e limitada, respectivamente, e $y_{0}=y_{1}(0)+y_{2}(0)$ a quantidade de carga total, no início do processo de descarga [7]. Os parâmetros $k^{\prime}, c$ e $y_{0}$ são estimados a partir de dados experimentais.

\section{Metodologia para Estimação dos Parâmetros}

Nesta seção é apresentada a estimação dos parâmetros $k^{\prime}, c$ e $y_{0}$, do Modelo KiBaM. Esses parâmetros são específicos para cada tipo de bateria, sendo que para os ensaios laboratoriais foram utilizadas 8 baterias novas de Li-Po, modelo PL-383562-2C. Os dados experimentais foram adquiridos a partir da plataforma de testes (i.e., testbed), desenvolvida por integrantes do GAIC [12].

Todas as coletas de dados seguiram a mesma metodologia, tanto para o carregamento das baterias, quanto para sua descarga. Para estimação dos parâmetros, foram utilizados 15 perfis de descarga constante, variando de $75 \mathrm{~mA}$ a $775 \mathrm{~mA}$, com intervalo entre perfis de $25 m A$. Cada perfil gerou, de modo individual, um tempo de descarga para cada bateria. A partir destes ensaios, foi possível calcular o tempo de vida médio para cada perfil.

Os MQ é um método de otimização que busca encontrar o valor ótimo dos parâmetros para um determinado conjunto de dados, através da minimização da soma dos quadrados dos resíduos. Este método é descrito pela seguinte equação

$$
V_{N}\left(\vec{\zeta}, Z^{N}\right)=\frac{1}{N} \sum_{t=1}^{N}(y(t)-\hat{y}(t \mid \zeta))^{2},
$$

onde: $\vec{\zeta}$ é um vetor que contém os parâmetros a serem estimados, $Z^{N}$ é um vetor que contém as entradas e saídas experimentais, $y(t)$ são as saídas experimentais medidas, $\hat{y}(t \mid \zeta)$ são as saídas calculadas a partir do modelo que contém os parâmetros que se deseja estimar, e $N$ é o número de dados experimentais [5].

As simulações foram implementadas com auxílio do software de computação algébrica e numérica MatLab, em uma rotina para a função de otimização lsqnonlin, que possui internamente a rotina dos MQ para problemas de otimização não linear. Os parâmetros encontrados para o Modelo KiBaM foram $k^{\prime}=37,46, c=0,01$ e $y_{0}=47356$.

\section{Análise dos Resultados}

Seguindo a mesma metodologia descrita na Seção 3, a partir de uma série de medições experimentais utilizando um smartphone, foram mensuradas as correntes em função de tarefas executadas pelo dispositivo. Deste modo, em seguida, definiu-se 8 perfis de descarga 
realísticos, com variações da corrente ao longo do tempo, conforme a Tabela 1. Ao contrário das descargas constantes, esses perfis possuem variações ao longo do tempo, assim, os efeitos não lineares têm maior presença e, por consequência, o processo de descarga torna-se mais fiel ao perfil de utilização de um usuário.

Tabela 1: Ciclo dos perfis de descarga variável.

\begin{tabular}{ccc}
\hline Perfil & Correntes $(m A)$ & Tempo $($ min $)$ \\
\hline P1 & $100-10-150-10-100-10-200$ & $5-5-5-5-5-5-10$ \\
P2 & $250-400-50-200-550$ & $10-10-5-15-10$ \\
P3 & $750-450-200-150-250-100$ & $5-10-10-5-5-10$ \\
P4 & $100-200-300-400-500-600-700$ & $10-10-10-10-10-10-10$ \\
P5 & $700-600-500-400-300-200-100$ & $10-10-10-10-10-10-10$ \\
P6 & $170-270-10-140-230-10-270$ & $5-20-30-10-20-10-30$ \\
P7 & $270-10-120-170-10-270-170$ & $5-10-10-15-10-15-5$ \\
P8 & $200-10-300-10-200-10-400$ & $2,5-5-2,5-5-2,5-5-5$ \\
\hline
\end{tabular}

Os experimentos produziram um conjunto de valores médios de tempo de vida, para cada um dos perfis, conforme a Tabela 2. O Modelo KiBam alcançou um erro médio de $1,84 \%$, sendo validado para predizer o tempo de vida de baterias de Li-Po. O Modelo $\mathrm{RV}^{6}$ aqui é utilizado para balizar os resultados em relação a outros modelos. Sabe-se que o Modelo RV possui acurácia reconhecida [9], assim, a partir dos resultados, é possível concluir que o Modelo KiBaM possui boa acurácia, sendo uma excelente alternativa para predizer o tempo de vida de baterias.

Tabela 2: Resultados das simulações.

\begin{tabular}{cccccc}
\hline \multirow{2}{*}{ Perfil } & \multirow{2}{*}{$L_{e}($ min $)$} & \multicolumn{2}{c}{ Modelo RV } & \multicolumn{2}{c}{ Modelo KiBaM } \\
& & $L_{s}($ min $)$ & Erro (\%) & $L_{s}($ min $)$ & Erro (\%) \\
\hline P1 & 479,68 & 479,38 & 0,06 & 478,38 & 0,27 \\
P2 & 149,38 & 148,53 & 0,57 & 148,53 & 0,57 \\
P3 & 141,76 & 144,17 & 1,70 & 144,18 & 1,71 \\
P4 & 126,62 & 122,9 & 2,94 & 122,87 & 2,96 \\
P5 & 98,51 & 98,35 & 0,16 & 98,32 & 0,19 \\
P6 & 284,94 & 269,48 & 5,43 & 269,15 & 5,54 \\
P7 & 322,01 & 331,55 & 2,96 & 330,27 & 2,57 \\
P8 & 324,17 & 327,58 & 1,05 & 327,00 & 0,87 \\
\hline \multicolumn{5}{c}{$E_{m}=1,86$} \\
\hline \multicolumn{5}{c}{}
\end{tabular}

\footnotetext{
${ }^{6}$ Este modelo foi utilizado como referência, contudo suas equações por fugirem do escopo do trabalho foram suprimidas. Entretanto, detalhamentos podem ser facilmente encontrados em [4], [9] e [10].
} 


\section{Conclusões}

Neste trabalho foi validado o Modelo KiBaM para predição do tempo de vida de baterias utilizadas em dispositivos móveis. Via de regra, trabalhos como este, empregam simuladores de baterias e perfis de descarga contínua. Contudo, nos experimentos conduzidos pelo GAIC, foram utilizadas 8 baterias novas de Li-Po e perfis de descarga variáveis. O uso de perfis variáveis busca emular o comportamento da bateria de um smartphone, de modo mais realístico, possibilitando que os efeitos não lineares fiquem mais evidentes.

A partir de um conjunto experimental de 8 perfis de descarga variável, foi realizada a simulação computacional do modelo obtendo um erro médio de $1,84 \%$, resultado inclusive ligeiramente melhor que do Modelo RV (1,86\%). Em perfis com menor possibilidade de ocorrer o efeito de recuperação (e.g., P4 e P6) os erros são maiores - o movimento é o contrário em perfis com maior capacidade de recuperação (e.g., P1 e P5). Isso aponta para o fato de que o modelo captura muito bem o efeito de taxa de capacidade, no entanto, há perdas significativas na modelagem do efeito de recuperação.

Em trabalhos futuros pretende-se aplicar os resultados obtidos no melhoramento do próprio modelo e de modelos híbridos, bem como, implementar computacionalmente o modelo estudado para gestão energética de dispositivos móveis.

\section{Agradecimentos}

À Coordenação de Aperfeiçoamento de Pessoal de Nível Superior (CAPES), pelo subsídio recebido, e à UNIJUÍ, pela infraestrutura disponibilizada.

\section{Referências}

[1] M. Doyle, T. Fuller, and J. Newmann. Modeling of galvanostatic charge and discharge of the lithium polymer insertion cell. Journal of Electrochemical Society, 140:15261533, jan. 1993.

[2] S. C. Hagemann. Simple PSpice models let you simulate common battery types. Electronic Design News, 38:117-129, 1993.

[3] C. F. Chiasserini and R. R. Rao. A model for battery pulsed discharge with recovery effect. In Wireless Communications and Networking Conference (WCNC '99), New Orleans, set. 1999.

[4] D. Rakhmatov and S. Vrudhula. An analytical high-level battery model for use in energy management of portable electronic systems. In Proceedings of the International Conference on Computer Aided Design (ICCAD '01), pages 488-493, Piscataway, 2001. IEEE Press.

[5] L. C. Romio. Modelagem matemática do tempo de vida de baterias utilizando a teoria de Identificação de Sistemas. Dissertação de mestrado, Universidade Regional do Noroeste do Estado do Rio Grande do Sul, Ijuí-RS, mar. 2013. 
[6] T. Kim. A hybrid battery model capable of capturing dynamic circuit characteristics an nonlinear capacity effects. Master's thesis, University of Nebraska, Lincoln-NE, jul. 2012 .

[7] J. Manwell and J. McGowan. Lead acid battery storage model for hybrid energy systems. Solar Energy, 50:399-405, mai. 1993.

[8] DUALFOIL. Fortran programs for the simulation of electrochemical systems. Disponível em: http://www.cchem.berkeley.edu/jsngrp/fortran.html. Acesso em: 10 ago. 2015.

[9] M. R. Jorgerden and B. Haverkort. Which battery model to use? Technical report, Imperial College London, 2008.

[10] D. J. B. Freitas. Estudo e aplicação de modelos analíticos na predição do tempo de vida de baterias utilizadas em dispositivos móveis: proposição de extensões aos modelos tradicionais. Dissertação de mestrado, Universidade Regional do Noroeste do Estado do Rio Grande do Sul, Ijuí-RS, set. 2015.

[11] GAIC. Aplicação de modelos matemáticos na predição do tempo de vida das baterias que alimentam dispositivos móveis. Disponível em: http://gaic.unijui.edu.br/ projdetalhes.php?id=3. Acesso em: 8 jan. 2015.

[12] H. B. Nonemacher, L. Minelli, P. S. Sausen, and A. Sausen. Desenvolvimento de um testbed para avaliação de modelos matemáticos utilizados na predição do tempo de vida de baterias. In Congresso Regional de Iniciação Científica e Tecnológica em Engenharia, 2010. 